\title{
Techno-economic and Simulation Study of a V2I-based Cooperative Manoeuvring Case in a Cross-border Scenario
}

\author{
A. Chiha Ep Harbi*, F. Poli ${ }^{\dagger}$, B. Denis ${ }^{\dagger}$, F. Vannieuwenborg*, D. Garcia-Roger ${ }^{\ddagger}$, J. F. Monserrat ${ }^{\ddagger}$ and W. Aigner $^{\S}$ \\ *IMEC-Ghent University, IDLab, Dept. of Information Technology, Technologiepark-Zwijnaarde 15, 9052 Ghent, Belgium \\ ${ }^{\dagger}$ CEA-Leti, Université Grenoble Alpes, F-38000 Grenoble, France \\ ‡Universitat Politècnica de València, ITEAM Research Institute, Camino de Vera s/n, 46022 Valencia, Spain \\ ${ }^{\S}$ HiTec, Lothringerstrasse 14/6, Vienna, Austria
}

\begin{abstract}
In this paper, we present a techno-economic analysis of a Cooperative, Connected and Automated Mobility (CCAM) use case in a specific cross-border environment, namely Cooperative Lane Merging (CLM). The latter is assumed to rely on Vehicle-to-Infrastructure (V2I) connectivity with respect to a set of inter-connected Road Side Units (RSUs). In order to feed the techno-economic framework with the required data in terms of road infrastructure, extensive system-level simulations have been performed using a connectivity oriented key performance indicator (KPI), while considering three different deployment scenarios and realistic road traffic densities. The proposed model identifies the minimum additional RSUs required to satisfy the CLM KPI with respect to the number of simultaneous connected cars. First results show the beneficial impact from densifying the road network infrastructure on the CLM service availability, especially under the highest road traffic conditions. In terms of Total Cost of Ownership (TCO), cost results of a set of scenarios considering the variation of both the number of connected cars and the RSUs to be deployed are discussed as well.
\end{abstract}

\section{INTRODUCTION}

The socio-economic need to develop mobility corridors at the continental scale, which has been emphasized by the Digital Single Market (DSM) policy of the European Commission [1], has been raising a variety of challenges on both technical and business sides. Major stakes typically concern e.g., the seamless cross-border continuity of Cooperative, Connected and Automated Mobility (CCAM) services (i.e., guaranteeing highly automated driving up to levels 4/5, according to the Society of Automotive Engineers' (SAE) classification), the massive adoption, the coexistence and the combination of various Vehicle-to-Everything (V2X) radio access technologies, the definition of supportive network architectures accordingly and finally, the deployment of (eventually service-specific) road or connectivity infrastructure [2]. In such cross-border contexts, the chain of both added values and costs is also complex to model, given the inherent multitenant nature of the ecosystem (including road operators, mobile network operators, cars and equipment manufacturers, service providers, institutional entities...). Key aspects of these technical and non-technical problems are currently addressed in the 5G-CARMEN project [3].
In this paper more specifically, as an illustration, we perform the techno-economic analysis of a Cooperative Lane Merging (CLM) use case in a representative cross-border highway context, while considering the support of Vehicleto-Infrastructure (V2I) connectivity with respect to Road Side Units (RSUs) based on the Cellular - V2X (C-V2X) sidelink (a.k.a., PC5-Mode4). For this purpose, we first introduce a generic Total Cost of Ownership (TCO) model, which has been developed to assess all the required costs over a specific period for the selected use case. This model integrates the Capital and Operational Expenditures (resp. CAPEX and OPEX), as well as additional overhead costs (e.g. marketing, helpdesk...). Conditioned upon distinct road infrastructure assumptions, these costs are subsequently mapped to the CLM service availability performances, under different penetration rates of connected cars and various road traffic conditions. The latter performances are evaluated by means of extensive simulations, using a connectivity-oriented key performance indicator (KPI) reflecting V2I multi-link reliability. Whereas most of recent simulation-based studies on C-V2X sidelink perform serviceagnostic evaluations of $\mathrm{V} 2 \mathrm{~V}$ communications at link level (e.g., [4]-[6]), we herein provide system-oriented evaluations (i.e., assuming an end-to-end multi-message information flow supporting a concrete off-loaded application), while putting the use of C-V2X sidelink in perspective with respect to road infrastructure deployment. One goal is thus to determine the best trade-offs between acceptable service availability and minimal RSUs deployment, depending on operating conditions. Beyond exploring the potential and the current limitations of PC5Mode4 aided V2I connectivity, this preliminary study also and foremost paves the way to new investigations regarding 5G deployment (including both Vehicle-to-Network (V2N) connectivity and Mobile Edge Cloud (MEC) considerations), while relying on a similar techno-economic evaluation framework. These side studies are also currently on-going in the 5G-CARMEN project. 


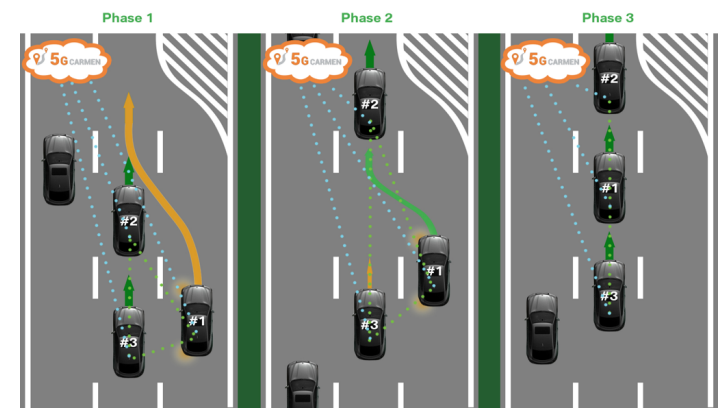

Fig. 1: Illustration of a 3-phase V2X-assisted CLM use case [7].

\section{Use CASE AND Working AsSUmptions}

\section{A. Cooperative Lane Merging Use Case}

We consider the reference CLM use case illustrated in Fig. 1 [7]. Accordingly, one vehicle intends to insert onto a side lane comprising already some vehicles. These side vehicles must hence create a gap, typically by slowing down or speeding up depending on their respective positions, so as to allow the initiator to insert between them. This manoeuvre is based on the prior collection of the positional information claimed by the different parties prior to the CLM execution (see Sec. II-C). In our specific operating context, the CLM procedure can be initiated and completed before, while or after crossing the border. The time elapsed between the initiator's request and the actual lane merge realization is hereafter referred to as the CLM negotiation phase.

\section{B. System Architecture and Deployment}

The information flow supporting the CLM negotiation relies on a semi-centralized ${ }^{1}$ operating mode, assuming V2I connectivity via PC5-Mode4 with respect to RSUs (see Fig. 2). These RSUs are assumed to be connected to a centralized computation-storage resource hosting the manoeuvre management application (e.g., Mobile Edge Cloud (MEC) or more generally, a server). The deployed RSUs can collect (resp. send) information from (resp. to) this centralized resource, which is in charge of making CLM decisions and notifications according to a "bird's eye view" (i.e., a vision of the scene elaborated to the best of all collected position awareness messages). In our cross-border study case between Italy and Austria, one RSU deployed close enough to the border on the Italian side, is expected to keep on collecting/broadcasting messages from/to vehicles located in a significant geographic area on the Austrian side, and reciprocally. However, besides addressing cross-border service continuity through direct coverage considerations, several RSUs deployed on both sides of the border (i.e., by two distinct national road operators) could also contribute to forge a common bird's eye view, while relying either on a shared/unique centralized resource or on distinct but coordinated centralized resources, assuming

\footnotetext{
${ }^{1}$ In opposition to a purely centralized configuration that would rely on V2N connectivity (i.e., via eNodeB/gNodeB base stations). Both V2Nbased centralized and V2V-based decentralized CLM modes are currently investigated in [3].
}

low-latency cross-border connections between them (i.e., if the decision is not made locally at the closest RSU).

\section{Messages Exchange Sequence}

In Fig. 3, we show the corresponding messages sequence chart [7], where default status messages are continuously broadcast by all the vehicles, including that involved in the CLM. These messages are collected by the RSUs and further forwarded to the MEC/server in charge of running the manoeuvre management application. At the beginning of the CLM negotiation phase, the initiator sends a request-to-merge message, which is also captured by the infrastructure and forwarded to the application. In return, based on the analysis of the latest received status messages (and thus, based on the relative positions of the involved vehicles), the application generates and transmits via the RSUs recommendation message(s) to the side vehicle(s) that must create the gap, as well as a safe-to-merge (or merge denial) message back to the initiator.

\section{Cost And Business Modeling of CLM in CROSS-BORDER SCENARIO}

\section{A. State of the Art}

Providing CCAM services in cross-border settings is not only challenging from a cost-effectiveness perspective but also from a business modelling one. In fact, deriving the cost of the required infrastructure to support CCAM provisioning deems crucial to judge the cost-effectiveness of such investment for both service and infrastructure providers. Yet, in a crossborder scenario it is not always clear who bears this cost and how the different stakeholders operating near the border, and sometimes under different laws and regulations, interact in order to guarantee a seamless service and connectivity continuity while crossing the border. These cost and business aspects need to be studied carefully to overcome the crossborder challenges. While several research papers can be found in literature regarding cost aspects from different angles e.g., socio-economic [8], cost-benefit analysis [9], business models for CCAM provision especially in cross-border environment are rarely investigated. Authors in [10] classified several papers based on various aspects, like communication, legislation, security and business. Among eight references, only one study partially investigated business aspects of V2X services. In this paper we will first study the cost required to deploy V2I infrastructure to support the CLM use case. Afterwards, insights into potential business models for CLM provision in cross-border scenarios will be discussed.

\section{B. Cost Model Description}

The proposed model consists of two main sub-models: the dimensioning process module and the cost model itself. First, key scenarios are specified, defining the outline of the network/system design principles. The latter then feeds the dimensioning process module, together with the existing infrastructure on the area under study, the size of this area, traffic densities, RSUs coverage and the time horizon. The dimensioning process thus digests all these inputs in order to 


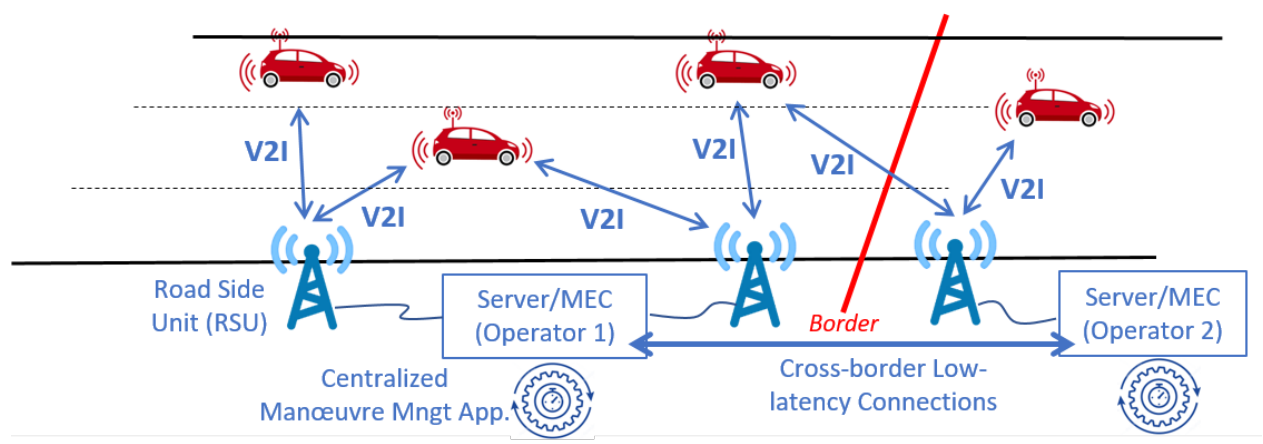

Fig. 2: Generic deployment and system architecture for semi-centralized CLM negotiations, where RSUs are connected to a MEC/server hosting the manoeuvre management application.

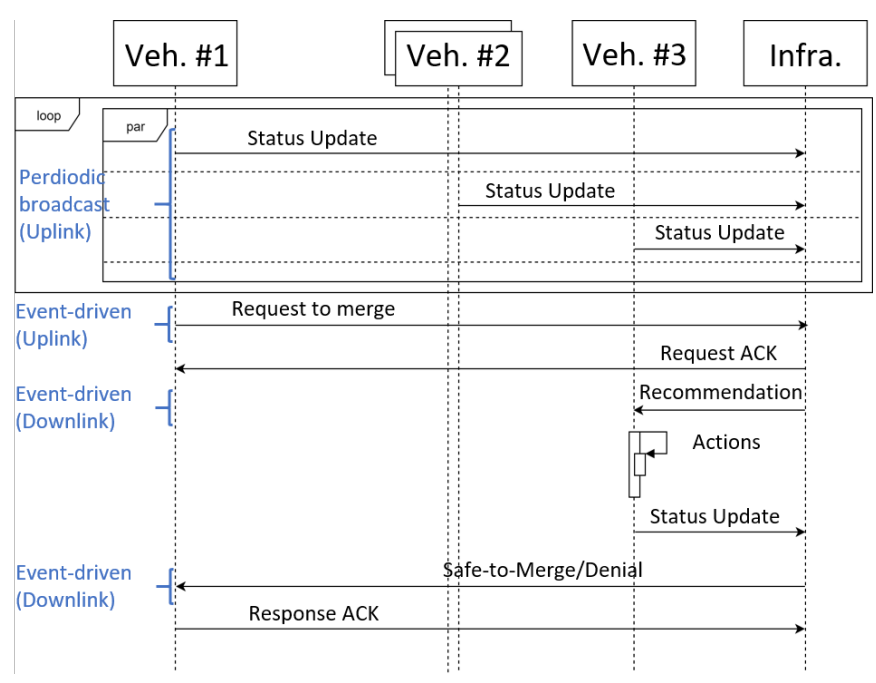

Fig. 3: Messages exchange sequence for V2I-based semi-centralized CLM [7].

find the minimum required infrastructure to cover the area of interest with regards to the defined KPIs, by means of simulations (See Sec. IV). The second main sub-model is the cost model. The main inputs are the Bill Of Materials (BOM), which is the main outcome of the dimensioning process, the element unitary cost, together with a clear distinction between CAPEX and OPEX elements. For our specific case, CAPEX incorporates the cost of RSUs and fibre and their installation cost. For the OPEX, it includes the power consumption of the installed RSUs, as well as the maintenance cost of the installed infrastructure i.e. RSUs and fibre. After calculating both CAPEX and OPEX, along with other overhead costs, the TCO can be derived. The generic structure of this model is presented in Fig. 4. Note that a cost allocation model can be used when evaluating the business case of a specific stakeholder together with revenue assumptions, which is out of scope in this paper.

\section{Cost Model Input and Assumptions}

We assume that the vehicles are equipped with standard hardware that can support default CCAM applications (e.g., manoeuvre engine...), On-Board Unit (OBU) components, V2X radio modem(s), and other required sensors. Therefore, the cost of equipping the cars is not taken into account here. We assume that the time horizon $T$ is 10 years (hence, no hardware renewal is anticipated since the lifetime of most equipment is about 10 years [11]). The yearly maintenance cost for V2I infrastructure is assumed to be $2 \%$ to $5 \%$ of the original hardware and labour costs (CAPEX). In addition, as the hardware installation cost cannot be found easily, hence we assume it to be $15 \%$ of the hardware costs [12]. In most cases, the overhead cost is defined as the cost of marketing, helpdesk, human resources, finance, etc. According to [13], it is around 20\% on top of the sum of CAPEX and OPEX. We assume also that CAPEX and OPEX cost inflation are respectively $-3 \%$ and $+3 \%$ per year [14]. Finally, as for the other cost data used in the study, they are confidential (i.e., project internal data), the retained figures fall in the following intervals: $2 \mathrm{k} €-5.5 \mathrm{k} €$ for the unitary cost per RSU, $10 \mathrm{k} €$ $15 \mathrm{k} €$ for the linear cost of $1 \mathrm{~km}$ of fibre (over the $4.5 \mathrm{~km}$ of highway considered in the simulations), $20 \mathrm{~W}-50 \mathrm{~W}$ for the average power consumption per RSU, assuming a $\mathrm{kWh}$ price of $10.85 €$-cents [15].

\section{Simulation FrameWORK}

For our simulation-based evaluations, we consider a systemlevel flow, which was initially developed in [16] and recently upgraded in [17], specifically to evaluate V2X-aided CLM negotiations. Accordingly, time-stamped mobility traces are generated offline using the Simulation of Urban MObility (SUMO) tool [18], feeding ns-3 network simulations ${ }^{2}$.

\section{A. Environment Description and Road Traffic Parameters}

The test environment consists of a $4.5 \mathrm{~km}$-long portion of the A22 highway located at the border between Italy and Austria, along with its secondary roads (e.g., the service lane SS12). This portion of highway comprises a $800 \mathrm{~m}$ long tunnel section, where we assume the presence of GPS repeaters to deliver the timing reference for PC5-Mode4 based communications.

\footnotetext{
${ }^{2}$ As we perform an information-centric evaluation of $\mathrm{V} 2 \mathrm{X}$ connectivity prior to CLM execution, mobility and connectivity simulations can remain decoupled (i.e., with no controlled feedback onto real-time vehicles' mobility).
} 


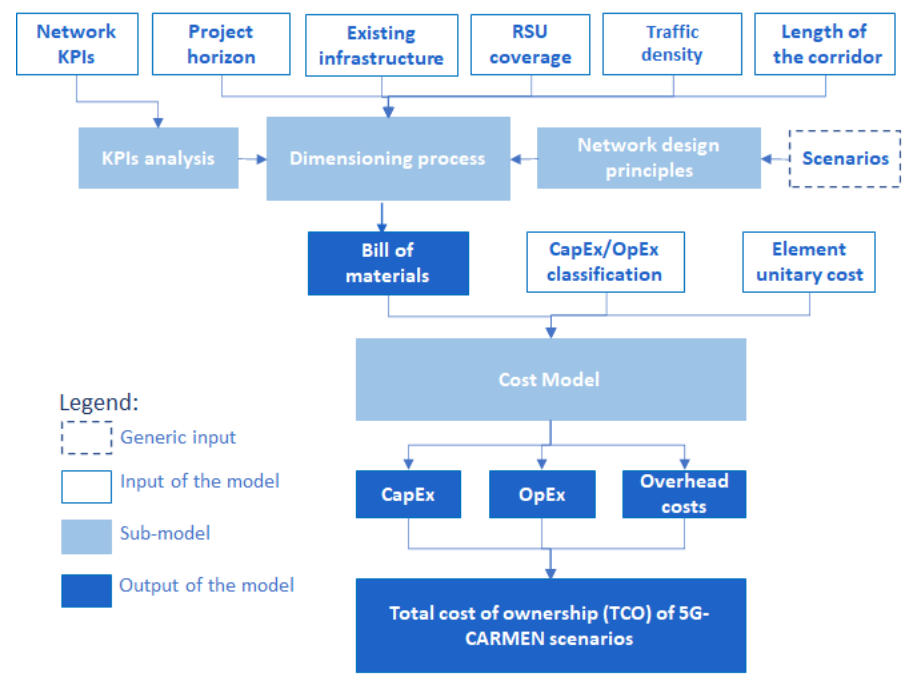

Fig. 4: Generic cost model structure.

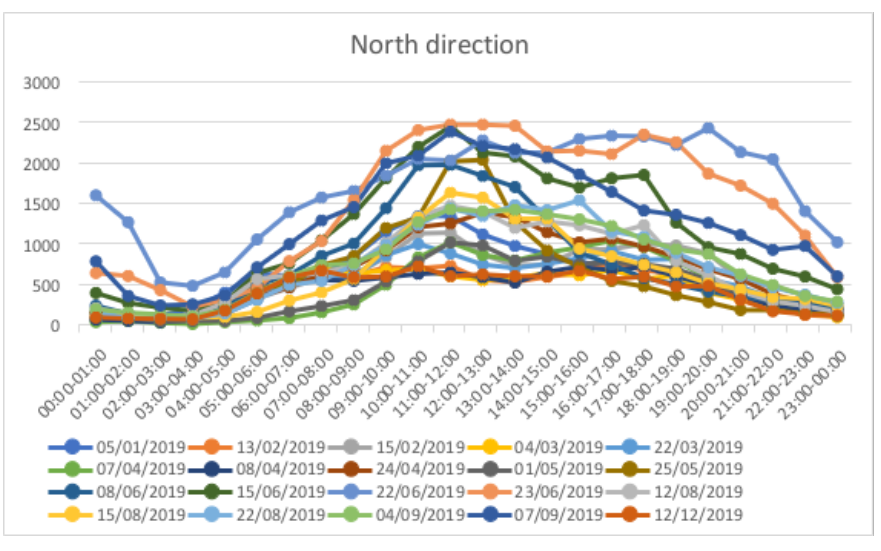

Fig. 5: Measured traffic (number of vehicles per hour) at the Brennero pass in the North driving directions, depending on both the period of the year and the hour of the day (Source: Autostrada del Brennero).

In this environment, real traffic measurements were collected in 2019 by Autostrada del Brennero at a reference point located $200 \mathrm{~m}$ away from the Italia-Austria border on the Italian side. This data set accounts for 5 days with maximum traffic volumes (critical traffic 100\%), 5 days in the third quartile (heavy traffic 75\%), 5 days in the median (smoothflowing traffic 50\%) and 5 days in the first quartile (low traffic volumes $25 \%$ ), considering the sum of the vehicles over the entire day on both carriage ways. It gives the count of vehicles in each lane of the motorway at the measurement point, where there are two lanes per direction, the data being aggregated per direction (See Fig. 5). These measurements reveal an upper (resp. lower) limit on the traffic flow in each driving direction at approximately 2500 (resp. 750) vehicles per hour over the busiest working hours (i.e., $9.00 \mathrm{am}-6.00 \mathrm{pm}$ ). This corresponds to an average traffic density of 83 (resp. 28) vehicles per kilometre.

The previous parameters have been used to configure the SUMO simulations based on macroscopic mobility parameters. In the latter simulations, $90 \%$ of the total amount of simulated vehicles were assumed to drive along the A22, without taking any service lane (i.e., staying in the A22 main loop). As a result, up to 90 "natural" lane merging situations involving 3 vehicles could hence be detected under the highest traffic density scenario, out of 3 minutes of a free-running SUMO simulation (i.e., $30 / \mathrm{min}$ ).

\section{B. Infrastructure Deployment}

The locations of the real-world RSUs physically deployed in the area of interest are represented with yellow markers on the right part of Fig. 6. Beyond, we have also considered increasing artificially the number of units to evaluate the beneficial impact of road infrastructure density on performance for different assumptions on the number of connected cars in the area under study (based on different market penetration rate of connected cars). This density varies from $0.5 \mathrm{RSUs} / \mathrm{km}$ (i.e., 2 RSUs over $4.5 \mathrm{~km}$; Scenario SC1), which corresponds to the current real-life baseline deployment (red locations on Fig. 6-left), to 2 RSUs $/ \mathrm{km}$ (i.e., 8 RSUs over $4.5 \mathrm{~km}$; Scenario SC3), quadrupling the baseline density (red plus yellow and blue locations on Fig. 6-left). One goal of the study is thus to determine the minimum road infrastructure density that could guarantee a typical service availability beyond $95 \%$, whatever the operating conditions.

\section{Radio Parameters}

The main radio parameters used for the ns- 3 simulation of PC5-Mode4 V2I links are similar to that in [17] (See Table I). We assume no packet segmentation but integral transmissions within time frames of $1 \mathrm{~ms}$ and a calibrated empirical 2slope log-normal average attenuation model [19], depending on (Obstructed) Line of Sight (OLoS) channel conditions. In terms of Medium Access Control (MAC), we consider a classical Sensing-Based Semi-Persistent Scheduling (SBSPS) algorithm, for which we have considered a selection window of $100 \mathrm{~ms}$. Knowing the Transmission Time Interval (TTI) occupancy of each single node, the Signal to Noise and Interference Ratio (SINR) per simulated link and accordingly, the average Packet Error Rate (PER), can thus be computed 


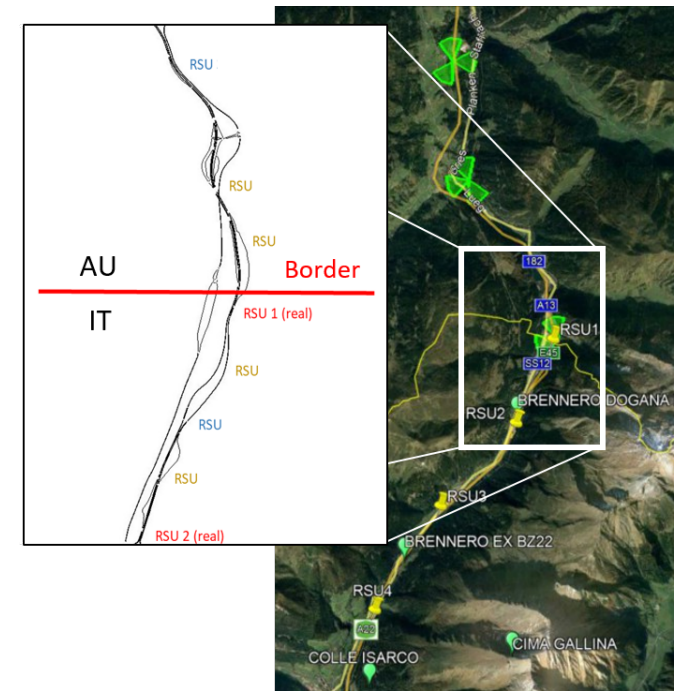

Fig. 6: Locations of RSUs considered in simulations, where the number of active RSUs vary from 2 [red] to 8 [red/blue/yellow].

TABLE I: Radio PHY parameters considered in PC5-Mode4 simulations

\begin{tabular}{|c|c|}
\hline Central frequency & $5.9 \mathrm{GHz}$ \\
\hline Transmit power & $23 \mathrm{dBm}$ \\
\hline Selection window & $100 \mathrm{TTIs}$ \\
\hline Reselection probability & 1 \\
\hline No. of RBs per Resource & 50 \\
\hline Messages size (status/others) & $200 / 125 \mathrm{bytes}$ \\
\hline No. of RBs for the TBs & 36 \\
\hline Modulation and coding scheme & $3 / 0$ \\
\hline 2-slope path loss exponents in LoS/OLoS & $\{-1.81,-2.85\} /\{-1.93,-2.74\}$ \\
\hline 2-slope path loss breakpoint distance & $100 \mathrm{~m}$ \\
\hline Reference path loss at 10 m in LoS/OLoS & $-63.9 /-72.3 \mathrm{~dB}$ \\
\hline Shadowing std. in LoS/OLoS & $4.15 / 6.67 \mathrm{~dB}$ \\
\hline
\end{tabular}

as a function of simulation time [17], [20] (e.g., physical layer PER performance vs. SINR can be derived from [4]). Accordingly, random realizations of packet reception/loss are drawn for each transmission attempt in the CLM messages sequence.

\section{Messages Formats and Rates}

Packet sizes of 125 bytes are assumed for on-demand request-to-merge, notification and safe-to-merge/denial messages. The communication from the application to the vehicles is based on Decentralized Environmental Notification Messages (DENMs) whereas Status update messages of 200 bytes are based on Cooperative Awareness Messages (CAMs).

Beside the few vehicles involved in the local CLM procedures under test, a gradually increasing proportion of the other vehicles belonging to the simulated flow (in first approximation, equivalent to the penetration rate of connected cars in the following) are assumed to transmit their own CAM messages at the same nominal average rate of $10 \mathrm{~Hz}$, while adapting their transmission parameters according to Decentralized Congestion Control (DCC) rules (e.g., reducing the transmission rate down to $2 \mathrm{~Hz}$ ), whenever the sensed instantaneous channel occupancy exceeds a threshold predefined by the standard [21].

\section{E. Connectivity-oriented Key Performance Indicator}

We evaluate the CLM negotiation availability from a pure connectivity perspective, as the capability to correctly receive all the messages requested in the negotiation phase, till the moment vehicles take actions. Concretely, in the absence of retransmissions, a semi-centralized negotiation is thus declared successful if the request message from the initiator vehicle is received by at least one of the RSUs (and then, forwarded to the CLM manoeuvre management application hosted in the MEC/server), the recommendation is received by the second vehicle in charge of creating the gap and the safe-to-merge (or denial) message from the infrastructure is received by the initiator.

Statistics are drawn over the total number of detected lane merging events, as well as over multiple random radio trials for each detected single lane merging event (typically, in terms of noise realizations, random access to the medium, etc.) to ensure sufficient diversity over both road traffic and radio dimensions, following a Monte Carlo approach.

\section{Results AND Discussions}

As both road and network operators usually deploy infrastructure to offer a bunch of services (rather than a unique service), it is neither straightforward, nor even realistic, to link directly the cost of a specific infrastructure deployment to a specific service provision without applying a cost allocation model, if the network resources can provide the minimum required service KPIs. Therefore, we run our cost model for different deployment scenarios aligned with the RSUs density assumptions of Sec. IV-B, which results in different values for the connectivity-oriented KPI assuming that $100 \%$ of the cars on the road are connected cars. In addition, this simulation was performed to give insights to road operators about the cumulative TCO if they want to replicate the existing infrastructure of the baseline scenario (i.e., 2RSUs) to another green field.

The cumulative TCOs of the three deployment scenarios, namely baseline/SC1, SC2 and SC3, are summarized in Table II. As expected, installing the first RSUs (baseline scenario/SC1) has a significant cost since fibre cable and electricity facilities need to be installed first, which are considered as the dominant investment cost component. Beyond, adding new RSUs will not have an important capital expenditure since the cabling to connect the additional RSUs is already present. The link between deployment scenarios and service feasibility can also be made based on the simulation results and more specifically, on the connectivity-oriented KPI described in Sec. IV-E. Overall, one can thus evaluate the nominal cost, in addition to the incremental cost of moving from one deployment scenario to the next, while making feasible (or not) the CLM service under upper and lower road traffic assumptions from Sec. IV-A. The corresponding results are summarized in Table II. One can thus clearly see the beneficial impact of densifying the road network infrastructure, especially under the highest road traffic conditions. Whereas a characteristic service availability threshold of $95 \%$ would not 
TABLE II: Joint evaluation of TCO and CLM negotiation availability vs. both road traffic and infrastructure densities assuming $100 \%$ penetration rate of connected cars.

\begin{tabular}{|c|c|c|c|}
\hline Infrastructure Density & TCO $(€)$ & Road Traffic Density & Service Availability $^{\mathrm{a}}$ \\
\hline \hline \multirow{2}{*}{$0.5 \mathrm{RSU} / \mathrm{km}$ (Baseline/SC1) } & \multirow{2}{*}{295,920} & $28 \mathrm{veh} . / \mathrm{km}$ & $95.8 \%$ \\
\cline { 3 - 4 } & & $83 \mathrm{veh} . / \mathrm{Km}$ & $71.9 \%$ \\
\hline \multirow{2}{*}{$1 \mathrm{RSU} / \mathrm{km}$ (SC2) } & 7,857 (Incremental $\left.^{\mathrm{b}}\right)$ & $28 \mathrm{veh} . / \mathrm{km}$ & $98.4 \%$ \\
\cline { 3 - 4 } & & $83 \mathrm{veh} . / \mathrm{Km}$ & $94.1 \%$ \\
\hline \multirow{2}{*}{$2 \mathrm{RSUs} / \mathrm{km}(\mathrm{SC} 3)$} & 10,284 (Incremental $\left.^{\mathrm{c}}\right)$ & $28 \mathrm{veh} . / \mathrm{km}$ & $93.9 \%$ \\
\cline { 3 - 4 } & & $83 \mathrm{veh} . / \mathrm{Km}$ & $98.7 \%$ \\
\hline
\end{tabular}
a Defined herein as the rate of completed CLM negotiations when all the required intermediary messages
have been correctly received.
b Additional cost comparing to the baseline scenario.
c Additional cost comparing to the second scenario.

TABLE III: The rate of rejected CLM requests based on the variation of the connected cars for the three infrastructure density scenarios

\begin{tabular}{|c|c|c|c|}
\hline Connected car penetration rate & SC1:2 RSUs & SC2:4 RSUs & SC3:8 RSUs \\
\hline $5 \%$ & $14.9 \%$ & $0 \%$ & $0 \%$ \\
\hline $10 \%$ & $17.1 \%$ & $3.4 \%$ & $0.1 \%$ \\
\hline $50 \%$ & $21.3 \%$ & $5.2 \%$ & $0.5 \%$ \\
\hline $100 \%$ & $28 \%$ & $6.4 \%$ & $0.7 \%$ \\
\hline
\end{tabular}

be reached at the system level within the current deployment setting of SC1 (and only hardly met after doubling the RSUs density up to $1 \mathrm{RSU} / \mathrm{km}$ in SC2), a density of $2 \mathrm{RSUs} / \mathrm{km}$ could fully restore CLM availability at some success rates comparable to that obtained under the lowest traffic conditions. Hence, a centralized application seems to clearly benefit from message redundancy over multiple links with respect to several RSUs simultaneously, whereas channel congestion can lead to the loss of critical messages over single links despite the use of DCC (i.e., while exceeding channel capacity). These results are somehow compliant with other independent studies performed at the link level, showing that an equivalent radio link reliability of $99 \%$ (required in our CLM case) would also be achieved under the same road infrastructure density of about $1 \mathrm{RSU} / \mathrm{km}$. However, it seems unrealistic to assume $100 \%$ of penetration rate of connected cars from the start. On the other hand, there is no common agreement within the research and concerned communities about convergent forecasts of this penetration rate in the upcoming years. There are relatively outdated studies with findings ranging from pessimistic [22] to optimistic [14] penetration rates, which cannot be used as such since the automotive industry was hardly hit by the Covid crisis [23]. Therefore, we decided to work with varying penetration rates, which are not linked to any specific starting year, i.e., working with abstracted years (year 1 to 10). Accordingly, we herein deliver generic results that road operators or CCAM providers could use and extrapolate whenever a clearer idea about the penetration rates and their evolution over time is made available. Hence, we re-run our simulation tool only for the high density SUMO trace (i.e., 83 veh./km), while varying the connected cars penetration rate for the three infrastructure densities (SC1: 2 RSUs, SC2: 4 RSUs and SC3: 8 RSUs). The corresponding simulation results, which are summarized in Table III, can be used to define a stepped deployment approach based on the defined service availability KPI. This approach assumes that road operators can work with 2RSUs (SC1) for 2 years (the max of penetration rate is $5 \%$ ), and then, as the penetration rate is approaching $10 \%$ by the third

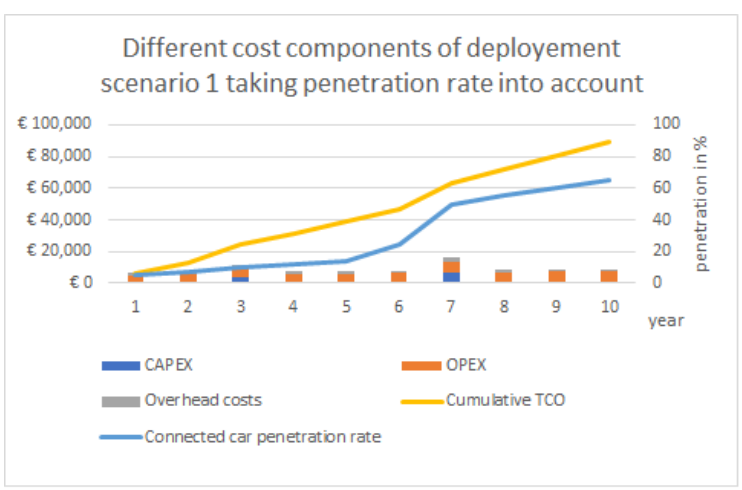

Fig. 7: Different cost components of the stepped approach for scenario 1: Currently deployed infrastructure at the Brenner Pass with respect to the penetration rate variation.

Different cost components of deployement scenario 2 taking penetration rate into account

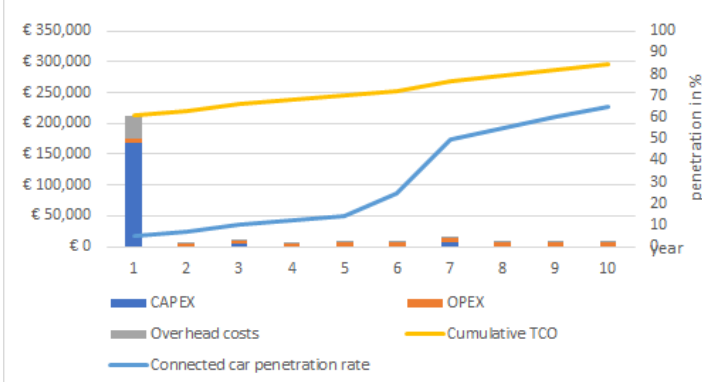

Fig. 8: Different cost components of the stepped approach for scenario 2:Green Field deployment with respect to the penetration rate variation.

year, 2 additional RSUs will thus be deployed to support the increase of traffic and be aligned with the defined KPI (i.e., CLM service availability). Afterwards and around year 7, the penetration rate is almost $50 \%$, hence 4 additional RSUs shall be installed to fulfill the defined KPI. This stepped approach is applied to two scenarios. The first one corresponds to the existing infrastructure of the Brenner pass, which implies that for the first two years only the OPEX is considered since no new RSUs are required. The second scenario corresponds to the duplication of the Brenner pass settings with the stepped deployment into a green field, where no fibre cables exist. Cost modelling results of the two scenarios are presented respectively in Fig. 7 and Fig. 8.

Similar to the previous analysis, if we have a green field the costs of equipping it with fibre and electricity cables make the deployment of the first RSUs costly in comparison with 
a pre-equipped field. Results of this stepped approach can give insights on how to cope with the increasing number of connected cars on the corridor in order to comply with the service KPI (related to CLM service availability here) while spreading the investment cost over time. From a business perspective, this deployment cost will not be paid by only one road operator, since the required additional RSUs will be deployed on the two sides of the border. This can cause an issue if the deployment strategies of the road operators near the border are not aligned. To solve this problem a collaboration between the different operators is needed. This collaboration can take up different forms ranging from agreement on deployment plans to active infrastructure sharing, where the two involved parties can share their infrastructure e.g., RSUs elements, fibre and MEC servers. The latter can solve the issue of service continuity in the cross-border area where a car that was connected to the RSU of operator A switches to the RSU of operator B after crossing the border line. This implies a switch to a new service/application instance, which can cause a serious problem if the car is in the process of executing the CLM manoeuvre. By having a shared MEC where a single application instance is running, this challenge can be overcome. MEC sharing models, as well as different collaboration business models, are subject of future work in [3].

\section{CONCLUSiOnS}

Considering a specific cross-border highway scenario, the techno-economic study accounted in this paper illustrates concrete trade-offs between the availability of a V2I-aided cooperative manoeuvring service (evaluated through system simulations as the success rate of completed multi-message CLM negotiations) and road infrastructure deployment costs, as a function of both road traffic and the number of connected cars. These evaluations show that a density of 2 RSUs $/ \mathrm{km}$ could be sufficient to achieve 95\% of CLM availability (as an a priori target), whatever the tested configurations. A stepped deployment approach was also studied aiming at providing more guidelines to road operators on how to cope with the increase of the number of connected cars in order to fulfill service KPIs while spreading the investment cost over time in a green field and pre-equipped context. Although the cross-border nature of the tested environment has only limited impact onto the previous simulation results (apart from realistic in-site traffic and propagation conditions), the latter suggest that V2N could be viably combined with such PC5Mode4 V2I links in case of local coverage shortage.

Still in the reference Brennero cross-border environment, side works currently on-going in [3] consider applying a similar techno-economic analysis framework to evaluate the potential of 5G-based V2N connectivity, while assuming different deployment assumptions for both MEC and gNodeB sites/sectors, as well as various time-wise hypotheses in terms of road traffic and service penetration. On this occasion, different variants of the use case shall also be considered (including in-lane manoeuvring scenarios, as well as other kinds/rates of messages, such as Cooperative Perception Messages (CPM)).

\section{ACKNOWLEDGMENT}

This work has been carried out in the frame of the $5 G$ CARMEN project [3], which is partly funded by the European Commission (H2020 GA No.825012).

\section{REFERENCES}

[1] "Digital Single Market program of the European Commission [Online]," https://ec.europa.eu/digital-single-market/en/, access: 2020-02-01.

[2] A. Kousaridas et al., " $5 \mathrm{G}$ Cross-Border Operation for Connected and Automated Mobility: Challenges and Solutions," Future Internet, vol. 12, no. $5,2020$.

[3] "5G-CARMEN [Online]," https://5gcarmen.eu/, access: 2020-02-01.

[4] V. Mannoni et al., "A Comparison of the V2X Communication Systems: ITS-G5 and C-V2X," in Proc. IEEE VTC'19-Spring, April 2019.

[5] D. Wang et al., "System-Level Simulator of LTE Sidelink C-V2X Communication for 5G," in Proc. ITG-Symposium on Mobile Communication - Technologies and Applications, May 2019.

[6] M. Gonzalez-Martín et al., "Analytical Models of the Performance of C-V2X Mode 4 Vehicular Communications," IEEE Trans. on Veh. Tech., vol. 68, no. 2, pp. 1155-1166, 2019.

[7] F. Visintainer et al., "5G CARMEN Use Cases and Requirements," Deliverable D2.1 of 5G-CARMEN project, May 2019.

[8] A. Raposo et al., "An analysis of possible socio-economic effects of a Cooperative, Connected and Automated Mobility (CCAM) in Europe," European Union Report, 2018.

[9] F. Ognissanto et al., "Investigation of the costs, benefits and funding models for two bundles of cooperative intelligent transport system services," IET Intelligent Transport Systems, vol. 13(6), pp. 1048-1056, 2019.

[10] M. Usman et al., "A Business and Legislative Perspective of V2X and Mobility Applications in 5G Networks," IEEE Access, vol. 8, pp. $67426-67435,2020$

[11] "U.S department of transportation Intelligent Transportation Systems Joint Program Office [Online]," https://www.itscosts.its.dot.gov/ITS/ benecost.nsf, access: 2020-02-01.

[12] K. Casier, "Techno-Economics of FTTH Deployment in the Presence of Competition," PhD Dissertation, Gent University, 2010.

[13] "A fitness program for Telcos [Online]," https://www.atkearney.com/ documents/20152/434204/Telco_Fitness.pdf.

[14] "Deliverable D2.3 Evaluation architecture design and socio-economic analysis - final report [Online]," http://www.it.uc3m.es/wnl/5gnorma/ pdf/5g_norma_d2-3.pdf.

[15] "Prices of electricity for the industry in Belgium from 2008 to 2018 [Online]," https://www.statista.com/statistics/595775, access: 2020-0201

[16] A. P. Da Silva et al., "System-level Simulation of Cooperative Sensor Data Fusion Strategies for Improved Vulnerable Road Users Safety," in Proc. IEEE WPNC'19, October 2019.

[17] F. Poli et al., "Evaluation of C-V2X Sidelink for Cooperative Lane Merging in a Cross-Border Highway Scenario," in Proc. IEEE VTCSpring'21, April 2021.

[18] D. Krajzewicz et al., "SUMO (Simulation of Urban MObility) - An Open-Source Traffic Simulation," in Proc. MESM'02, Sept. 2002.

[19] T. Abbas et al., "A Measurement Based Shadow Fading Model for Vehicle-to-Vehicle Network Simulations," Int. Journal of Ant. and Propag., vol. 2015, 2015.

[20] A. Guizar et al., "LTE-V2X Performance Evaluation for Cooperative Collision Avoidance (CoCA) Systems," in Proc. IEEE VTC-Fall'20, Oct. 2020.

[21] A. Bazzi, "Congestion Control Mechanisms in IEEE 802.11p and Sidelink C-V2X," in Proc. IEEE Asilomar'19, Nov. 2019.

[22] "D2.1 Vehicle fleet penetrations and ODD coverage of NRA relevant automation functions up to 2040 [Online]," https://www.mantra-research. eu/wp-content/uploads/2020/03/MANTRA_Deliverable_D2.1_1.0.pdf.

[23] "COVID-19: Automotive industry signs joint Code of Business Conduct to support re-start of production [Online]," https://www.acea.be/ press-releases/article/covid-19-automotive. 